\title{
PGC7, H3K9me2 and Tet3: regulators of DNA methylation in zygotes
}

\author{
Jinsuk Kang ${ }^{1}$, Sundeep Kalantry ${ }^{2}$, Anjana Rao ${ }^{1}$ \\ ${ }^{1}$ La Jolla Institute for Allergy and Immunology, La Jolla, CA 92037, USA; ${ }^{2}$ Department of Human Genetics, University of Michi- \\ gan Medical School, Ann Arbor, MI 48109, USA \\ Cell Research (2013) 23:6-9. doi:10.1038/cr.2012.117; published online 7 August 2012
}

In zygotes, a global loss of DNA methylation occurs selectively in the paternal pronucleus before the first cell division, concomitantly with the appearance of modified forms of 5-methylcytosine. The adjacent maternal pronucleus and certain paternallyimprinted loci are protected from this process. Nakamura et al. recently clarified the molecular mechanism involved: PGC7/Stella/Dppa3 binds to dimethylated histone 3 lysine 9 (H3K9me2), thereby blocking the activity of the Tet 3 methylcytosine oxidase in the maternal genome as well as at certain imprinted loci in the paternal genome.

DNA methylation is a crucial epigenetic modification that regulates imprinting (differential silencing of maternal or paternal alleles) and repression of retrotransposons and other parasitic DNA, as well as possibly $\mathrm{X}$-chromosome inactivation and cellular differentiation. DNA methylation needs to be faithfully maintained throughout the life cycle, since loss of DNA methylation can result in gene dosage problems, dysregulation of gene expression, and genomic instability due to retrotransposon reactivation [1]. Nevertheless, genome-wide loss of

Correspondence: Anjana Rao

E-mail: arao@liai.org
DNA methylation has been observed during germ cell development [2] and in the paternal pronucleus soon after fertilization [3].

For almost a decade, the global decrease of DNA methylation observed in the paternal genome within a few hours of fertilization was ascribed to an "active", replication-independent process [3]. The maternal pronucleus is spared and instead undergoes "passive", replication-dependent demethylation during early embryogenesis, arising from inhibition of the DNA maintenance methyltransferase Dnmt1 (Dnmt1 is normally recruited to newlyreplicated DNA because of the high affinity of its obligate partner, UHRF1, for hemi-methylated DNA strands, which are produced from symmetricallymethylated $\mathrm{CpG}$ dinucleotides as a result of DNA replication). The basis for active and passive demethylation of the paternal and maternal genomes remained a mystery until proteins of the TET family - TET1, TET2 and TET3 in humans - were discovered to be Fe(II)and 2-oxoglutarate-dependent enzymes capable of oxidizing 5-methylcytosine $(5 \mathrm{mC})$ in DNA [4-6]. TET enzymes serially convert $5 \mathrm{mC}$ into 5 -hydroxymethyl-cytosine $(5 \mathrm{hmC}), 5$-formylcytosine $(5 \mathrm{fC})$ and 5 -carboxycytosine $(5 \mathrm{caC})$ $[5,7,8]$.

With the generation of specific antibodies to $5 \mathrm{hmC}$, it became clear that the supposed "active demethylation" of the paternal pronucleus in mouse zygote after fertilization was due to the inability of anti-5mC antibodies to recognize $5 \mathrm{hmC}$ and other $5 \mathrm{mC}$ oxidation products $[9,10]$. The enzyme responsible for $5 \mathrm{mC}$ oxidation was shown to be Tet3, which unlike Tet1 and Tet2 is highly expressed in mouse oocytes and zygotes. RNAi-mediated depletion of Tet 3 decreased the staining of the paternal pronucleus with $5 \mathrm{hmC}$, suggesting that immediately after fertilization, Tet3 in the zygote selectively oxidizes $5 \mathrm{mC}$ in the paternal genome to $5 \mathrm{hmC}[9,10]$.

How is the maternal pronucleus protected from Tet3 activity? Nakamura et al. [11] previously showed that zygotes lacking PGC7/Stella/Dppa3 lose asymmetric regulation of DNA methylation, instead showing global loss of $5 \mathrm{mC}$ staining in both paternal and maternal pronuclei. This was correlated with hypomethylation at several maternally-imprinted loci (Peg1, Peg3, $P e g 10)$ in $P G C 7$-deficient zygotes, as judged by bisulfite sequencing. Further, certain paternally-imprinted loci (H19, Rasgrf1), which are normally protected from global loss of methylation in the paternal genome, also became hypomethylated in $P G C 7$-deficient zygotes. These data suggested that PGC7 protects the maternal genome, as well as certain paternally imprinted loci, from loss of $5 \mathrm{mC}$. 
In their recent publication, Nakamura et al. [12] elegantly extended these findings to address the mechanism involved. Based on the fact that a major difference between maternal and paternal genomes is that the maternal genome contains histones, whereas the DNA of the entering sperm is tightly packaged with protamine, they asked whether PGC7 recognizes specific histone marks. Indeed, the maternal genome harbors considerable levels of the histone mark H3K9me2 [11], leading them to examine whether PGC7 distinguishes maternal and paternal genomes by recognizing $\mathrm{H} 3 \mathrm{~K} 9 \mathrm{me} 2$ in the maternal genome. Using wild-type (WT) ES cells and ES cells deficient in the G9a lysine methyltransferase which generates $\mathrm{H} 3 \mathrm{~K} 9 \mathrm{me} 2$ mark, they showed that PGC7 associated loosely with nucleosomes and chromatin lacking $\mathrm{H} 3 \mathrm{~K} 9 \mathrm{me} 2$, but tightly if $\mathrm{H} 3 \mathrm{~K} 9 \mathrm{me} 2$ was present. The binding was recapitulated using recombinant bacterially-expressed PGC7 and histone tail peptides, indicating a direct interaction of $\mathrm{PGC} 7$ with the $\mathrm{H} 3 \mathrm{~K} 9 \mathrm{me} 2$ mark. In agreement, genomic loci enriched with $\mathrm{H} 3 \mathrm{~K} 9 \mathrm{me} 2$ recruited $\mathrm{PGC7}$ as judged by chromatin immunoprecipitation (ChIP), but this recruitment was abrogated in $G 9 a$ deficient ES cells. These data indicated that PGC7 targets genomic regions occupied by nucleosomes containing H3K9me2 (Figure 1); an interesting extension would be to ask whether loss of maternal $G 9 a$ also results in $5 \mathrm{hmC}$ conversion in the maternal pronucleus in zygotes.

Next, Nakamura et al. [12] tested by immunocytochemistry whether PGC7 in zygotes also required $\mathrm{H} 3 \mathrm{~K} 9 \mathrm{me} 2$. It is known that $\mathrm{H} 3 \mathrm{~K} 9 \mathrm{me} 2$ staining is concentrated in the maternal but not the paternal pronucleus [13]. Using conventional staining methods in which the cells are first fixed and then permeabilized to allow antibodies to enter the cell, the authors observed in their earlier study that PGC7 bound to both pronuclei [11]. Remarkably, by simply reversing the order of the fixation and permeabilization steps - permeabilizing first to allow the loss of loosely bound proteins by dissociation, then fixing and staining - they found that PGC7 associated much more tightly with the maternal pronucleus that bears $\mathrm{H} 3 \mathrm{~K} 9 \mathrm{me} 2$ mark. Injection of mRNA encoding Jhdm2a (an H3K9me1/ me2-specific demethylase) into zygotes eliminated staining for $\mathrm{H} 3 \mathrm{~K} 9 \mathrm{me} 2$ as well as PGC7 in the maternal pronucleus, and concomitantly caused loss of $5 \mathrm{mC}$ and acquisition of $5 \mathrm{hmC}$. Taken together, these data strongly suggested that PGC7 was selectively recruited to the maternal pronucleus through binding $\mathrm{H} 3 \mathrm{~K} 9 \mathrm{me} 2$, and that this binding protected zygotic maternal DNA from oxidation of $5 \mathrm{mC}$ to $5 \mathrm{hmC}$ and beyond (Figure 1).

These findings led Nakamura et al. to investigate how PGC7 controls Tet3 activity in zygotes. They showed (in cells that were permeabilized before fixation and immunocytochemistry) that Tet 3 was tightly associated only with the paternal pronucleus in WT zygotes, but was present in both pronuclei in $P G C 7$ deficient zygotes. When PGC7 was prevented from binding to the maternal pronucleus by injection of $J h d m 2 a$ mRNA, Tet3 became tightly associated with

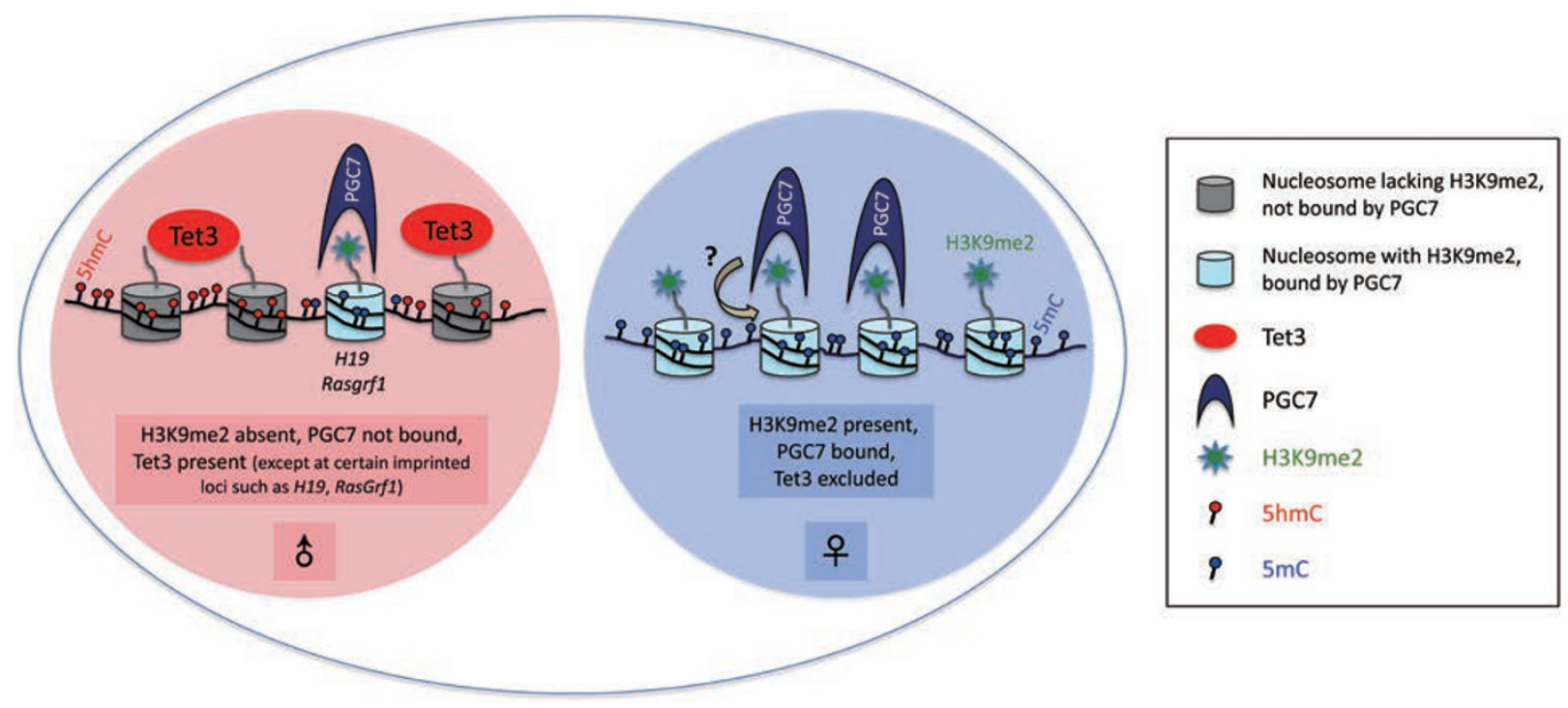

Figure 1 Schematic view of paternal (left) and maternal (right) genomes soon after fertilization. Paternal and maternal pronuclei are indicated with immunostaining results in the boxes. PGC7 binds H3K9me2 in the maternal pronucleus and at certain paternally-imprinted loci (H19, Rasgrf1) in the paternal pronucleus, thereby potentially regulating chromatin organization to interfere with Tet3 accessibility. 
both pronuclei. In other words, loss of PGC7 or loss of H3K9me2 that recruits PGC7 had the same effect - eliminating selective association of Tet 3 with the paternal genome. The implication is that PGC7 - which preferentially binds the maternal genome - somehow promotes the selective binding of Tet3 to the paternal genome, thus permitting rapid $5 \mathrm{mC}$ oxidation in paternal but not maternal DNA (Figure 1).

PGC7 is a small protein (150 amino acids (aa) in the mouse, 159 aa in humans) whose sequence is only moderately conserved. Nakamura et al. [12] showed that the binding of PGC7 to H3K9me 2 required the $\mathrm{N}$-terminal half of PGC7, whereas its ability to exclude Tet3 from the maternal pronucleus required the $\mathrm{C}$-terminal half. It is unclear how Tet3 exclusion is mediated. One possibility is that the $\mathrm{C}$-terminal region of PGC7 sterically excludes Tet3 from binding, either to DNA or to a chromatin mark; another is that the C-terminal region of $\mathrm{PGC7}$ is capable of altering chromatin configuration to prevent the binding of Tet 3 to chromatin. In support of the latter hypothesis, the rate with which micrococcal nuclease (MNase) digested high-molecular weight chromatin was significantly slower in WT ES cells in which PGC7 was present, compared to $\mathrm{PGC7}^{-/-}$and $G 9 a^{-/-} \mathrm{ES}$ cells in which PGC7 was either absent or not recruited to DNA because of the loss of H3K9me2 mark. In contrast, DNA methylation did not alter the chromatin association of $\mathrm{PGC7}$ or its ability to protect high-molecular weight chromatin from MNase digestion, as shown by using Dnmt $1^{-/}$Dnmt $3 a^{-/}$Dnmt $3 b^{-/-}$ triple knockout ES cells that completely lack DNA methylation.

How does PGC7 protect paternallyimprinted loci from Tet3-mediated $5 \mathrm{mC}$ oxidation? Although the haploid sperm genome is mostly packaged with protamine, a genome-wide analysis revealed that $4 \%$ of the genome of mature human sperm bears nucleosomes located at developmental and imprinted genes [14]. Nakamura et al. [12] found that among paternally-imprinted differentially methylated regions (DMRs), the H19 and Rasgrfl DMRs contained H3K9me2 whereas the Meg3 DMR did not, consistent with their previous finding that in $P G C 7$-deficient zygotes, the H19 and Rasgrf1 DMRs were hypomethylated but the Meg3 DMR was unaffected [11]. Therefore, PGC7 may be recruited to paternally-imprinted loci through $\mathrm{H} 3 \mathrm{~K} 9 \mathrm{me} 2$-containing nucleosomes that pre-exist in the sperm haploid genome upon fertilization. Alternatively, Nakamura et al. point out that protamine in the sperm is replaced soon after fertilization by the histone H3.3 variant, which in somatic cells does not bear $\mathrm{H} 3 \mathrm{~K} 9 \mathrm{me} 2$ mark.

In conclusion, Nakamura et al. [12] demonstrate unambiguously that PGC7 specifically binds to H3K9me2 in the maternal genome in zygotes, where its global occupancy excludes Tet 3 and inhibits Tet3-mediated $5 \mathrm{mC}$ oxidation. This novel finding provides new insights into the global alterations of DNA methylation status that occur during early embryogenesis. Followup questions abound. First, can PGC7 protect other methylated loci such as transposable elements and the $\mathrm{X}$ chromosome? It would be interesting to assess H3K9me2 at these loci. Second, how does the N-terminal half of PGC7 recognize H3K9me2? Structural characterization of this interaction may elucidate a novel epigenetic "reader" domain specific for $\mathrm{H} 3 \mathrm{~K} 9 \mathrm{me} 2$. Third, $\mathrm{PGC} 7$ is a marker for cells of the inner cell mass, and is co-expressed with Tet1 and Tet2 rather than Tet3 in ESCs [15]. Does PGC7 also antagonize Tet 1 and Tet 2 and protect imprinted loci in ESCs? Fourth, how does PGC7 inhibit the access of Tet 3 to chromatin? Considering that $\mathrm{PGC7}$ is small and is not equipped with known enzymatic domains, it is likely that PGC-interacting proteins, rather than PGC7 itself, function to regulate chromatin status. Fifth, how is Tet3 recruited to paternal chromatin - are there specific histone or other epigenetic marks that facilitate Tet3 recruitment? Finally, while technically challenging, it seems imperative to identify the target genes of PGC7 and Tet3, by profiling the genomic location of $5 \mathrm{hmC}$ and other $5 \mathrm{mC}$ oxidation products in the paternal and maternal genomes of zygotes from WT, Tet3-deficient and PGC7-deficient mice.

\section{Acknowledgments}

This work was supported by NIH grant HD065812 and grant RM1-01729 from the California Institute of Regenerative Medicine (to AR), and NIH grants R00HD055333 and DP2-OD-008646 and grants from the Ellison Medical Foundation and March of Dimes to SK. JK is supported by a postdoctoral fellowship from the Jane Coffin Childs Memorial Fund for Medical Research.

\section{References}

1 Ooi SK, O’Donnell AH, Bestor TH. Mammalian cytosine methylation at a glance. J Cell Sci 2009; 122:27872791.

2 Hackett JA, Zylicz JJ, Surani MA. Parallel mechanisms of epigenetic reprogramming in the germline. Trends Genet 2012; 28:164-174.

3 Mayer W, Niveleau A, Walter J, Fundele R, Haaf T. Demethylation of the zygotic paternal genome. Nature 2000; 403:501-502.

4 Iyer LM, Anantharaman V, Wolf MY, Aravind L. Comparative genomics of transcription factors and chromatin proteins in parasitic protists and other eukaryotes. Int J Parasitol 2008; 38:131.

5 Tahiliani M, Koh KP, Shen Y, et al. Conversion of 5-methylcytosine to 5-hydroxymethylcytosine in mammalian DNA by MLL partner TET1. Science 2009; 324:930-935.

6 Iyer LM, Tahiliani M, Rao A, Aravind L. Prediction of novel families of enzymes involved in oxidative and other complex modifications of bases in nucleic acids. Cell Cycle 2009; 8:16981710.

7 Ito S, Shen L, Dai Q, et al. Tet proteins can convert 5-methylcytosine to 
5-formylcytosine and 5-carboxylcytosine. Science 2011; 333:1300-1303.

8 He YF, Li BZ, Li Z, et al. Tet-mediated formation of 5-carboxylcytosine and its excision by TDG in mammalian DNA. Science 2011; 333:1303-1307.

9 Iqbal K, Jin SG, Pfeifer GP, Szabó PE. Reprogramming of the paternal genome upon fertilization involves genome-wide oxidation of 5-methylcytosine. Proc Natl Acad Sci USA 2011; 108:3642-3647.

10 Wossidlo M, Nakamura T, Lepikhov K, et al. 5-Hydroxymethylcytosine in the mammalian zygote is linked with epigenetic reprogramming. Nat Commun 2011; 2:241.

11 Nakamura T, Arai Y, Umehara H, et al. PGC7/Stella protects against DNA demethylation in early embryogenesis. Nat Cell Biol 2007; 9:64-71.

12 Nakamura T, Liu YJ, Nakashima H, et al. PGC7 binds histone $\mathrm{H} 3 \mathrm{~K} 9 \mathrm{me} 2$ to protect against conversion of $5 \mathrm{mC}$ to $5 \mathrm{hmC}$ in early embryos. Nature 2012; 486:415-419.

13 Santos F, Peters AH, Otte AP, Reik W, Dean W. Dynamic chromatin modifi- cations characterise the first cell cycle in mouse embryos. Dev Biol 2005; 280:225-236.

14 Hammoud SS, Nix DA, Zhang H, Purwar J, Carrell DT, Cairns BR. Distinctive chromatin in human sperm packages genes for embryo development. Nature 2009; 460:473-478.

15 Hayashi K, Lopes SM, Tang F, Surani MA. Dynamic equilibrium and heterogeneity of mouse pluripotent stem cells with distinct functional and epigenetic states. Cell Stem Cell 2008; 3:391-401. 